\title{
Cellulose / Copper Blend and Porous Copper Prepared by Mechano-Chemical Treatments
}

\author{
Hitomi Miyamoto, Chisato Ueda, Chihiro Yamane, Mariko Ago*, Kunihiko \\ Okajima*, Takashi Endo**, Makiko Hattori*** \\ Faculty of Home Economics, Kobe Women's University, Aoyama 2-1, Higashisuma Suma-ku, Kobe \\ 654-8585, Japan \\ FAX:81-78-732-5161,e-mail: yamane@suma.kobe-wu.ac.jp \\ *Faculty of Engineering, Tokushima Bunri University, Shido 1314-1, Sanuki-shi, Kagawa 100-0004, \\ Japan \\ FAX:81-87-894-4201,e-mail: okajima@fe.bunri-u.ac.jp \\ ** Advanced Industrial Science and Technology, 2-2-2, Hiro-suehiro Kure, Hiroshima 737-0197 \\ Japan \\ FAX:81-823-72-1990, e-mail: t-endo@aist.go.jp \\ *** Central R\&D Laboratories, Asahi Kasei Corporation, 2-1, Samejima, Fuji, Shizuoka, 416-8501, \\ Japan \\ FAX:81-545-62-3179, e-mail: hattori.mb@om.asahi-kasei.co.jp
}

A cellulose/copper blend was prepared by a mechano-chemical treatment using a high-speed ball mill called a planetary mill. Cellulose and copper in the blend were mixed in the submicron scale, as observed by scanning probe microscopy using the surface potential microscopic mode. The treatment made the cellulose crystal almost amorphous while the molecular weight of the cellulose retained $70 \%$ of its original value. X-ray diffraction patterns of the copper remained unchanged. Removal of cellulose from the blend by firing or enzymatic decomposition yielded porous copper, the surface area of which was $18 \mathrm{~m}^{2} / \mathrm{g}$. The copper fragments appeared not to be isolated but rather were connected to each other at junction points. Such a porous structure must prevent cohesion of each metal fragment to retain its effective surface area. Many nano-metals have a problem, in that the cohesion of the particles decreases their apparent surface area resulting in a decrease in the efficiency for their practical use. Therefore, if porous metals prepared by this method are applied as catalysts or electrodes, a high efficiency can be expected.

Key words: cellulose, mechano-chemical, blend, metals, composite

\section{INTRODUCTION}

Mechanical alloying has been widely studied for many materials, such as metal alloys and polymer blends, because of the wide range of its applicability. Conventional polymer blends are mainly prepared from the melt mixtures of the polymers or solutions, which require the polymers to be melts or co-solvents of them. Cellulose is thus not suitable as a material for such blendings because it can not melt under the temperature of thermal decomposition and has few co-solvents with commonly used polymers. Mechanical alloying however has been used for forming blends whose components are not chemically miscible with each other. Blends of cellulose and thermoplastic polymers were recently prepared for the first time by mechano-chemical treatment using a high-speed ball mill called a planetary mill ${ }^{1,2}$. By the treatment, the blends with cellulose content over $80 \%$ were thermally flow-able as to be applied to injection molding. It is to be noted that blends mixed by a conventional extruder cannot be shaped by injection molding. In this context, mechanical alloying offers a new avenue for preparing polymer/metal blends with fine dispersion.

Recently, polymer/metal nano-composites have been prepared using various methods, including (1) evaporation of metals on a polymer surface followed by dispersion into the polymer by molecular movement of the polymer segments above the glass transition temperature ${ }^{3,4} ;$ (2) independently evaporating organic and metallic raw materials and thereby covering the metal with the organic material ${ }^{5}$; (3) selective localization of metal particles in a polymer domain using a micro-phase separation method $^{6}$; (4) absorption of hydrophobic parts of polymers on a metal surface forming a polymer/metal composite in order to prevent aggregation of these metals ${ }^{7}$. Various additional studies relating to polymer/metal nano-composites have been conducted, and a complete review is beyond the scope of this paper. However, it is to be noted that mechanical alloying has not been attempted thus far to prepare polymer/metal composites. 
In this study, we prepared cellulose/copper blends using a planetary mill and examined the structure of the blends. In addition, porous copper prepared by removing cellulose from the blend by firing or enzymatic decomposition was also investigated.

\section{EXPERIMENTAL}

2.1 Blend of Copper and Cellulose

Copper powder, under $80 \mu \mathrm{m}$ in diameter, prepared using an atomizing method was purchased from Kojundo Chemical Laboratory Co Ltd., Japan. Cellulose powder supplied by Whatman International Ltd., England, was used as the cellulose sample; the sample name was CF-11. Fourteen grams of copper, $6 \mathrm{~g}$ of cellulose and 25 zirconia balls $(20-\mathrm{mm}$ diameter) were placed in a zirconia vessel with a $500 \mathrm{ml}$ volume. The vessel was set in the high-speed ball mill (Model P-5, Fritsch GmbH, Germany) and subjected to planetary motion capable of moving the balls with a high acceleration over 10 times the gravitational acceleration. The copper and cellulose mixture was ball milled using this apparatus at $250 \mathrm{rpm}$, which is equivalent to 10 times the acceleration of gravity, at under $80^{\circ} \mathrm{C}$ for given times. This treatment was conducted intermittently, 30 minutes running followed by a 15 minute pause, such that the sample temperature did not exceed $80^{\circ} \mathrm{C}$.

2.2 Preparations of Porous Copper

Porous copper was prepared by removing cellulose from the blend. Two methods were employed to remove cellulose. In the first method, the copper and cellulose blend was fired at $600^{\circ} \mathrm{C}$ for 3 hours to remove the cellulose. In the second method, the copper and cellulose blend was treated with 1 wt\% aq. cellulase (Onozuka R-10 derived from Trichoderma viride, Yakult Co. Ltd., Japan) at $50^{\circ} \mathrm{C}$ and at a $\mathrm{pH}$ of 4.5 in an acetic acid buffer $(0.1 \mathrm{~N}$ acetic acid $/ 0.1 \mathrm{~N}$ sodium acetate $=10 / 7$ $\mathrm{v} / \mathrm{v})$ for one week. Complete removal of cellulose from the blend was confirmed by the weight loss of the sample, equal to the cellulose content in the blend.

2.3 Measurements

Scanning Electron Microscopy (SEM): The blend of copper and cellulose was observed using a S-4700 (Hitachi, Ltd.) SEM operated at an accelerating voltage of $15 \mathrm{kV}$. This microscope is also equipped with an energy dispersive X-ray micro analyzer (EMAX-7000, HORIBA, Ltd.).

Scanning Probe Microscopy (SPM): The blend sample was placed on a stainless steel sample holder covered with carbon paste, and observed with a scanning probe microscope (Nano Scope IV D3100 with $\mathrm{Cr} / \mathrm{Au}$ coated probes named MESP, Veeco Instruments Inc.) using a surface potential microscopic mode (SPoM).

Wide-Angle X-ray Diffraction: X-ray diffraction patterns were measured using a reflection method and recorded on an X-ray diffraction apparatus with a scintillation counter (Rotaflex Ru-200PL, Rigaku Denki Co. Ltd., Japan) with an inner reference $\left(\mathrm{SiO}_{2} ; 2 \theta=28.45^{\circ}\right)$.

Surface Area: The surface area of the porous copper was measured by the amount of nitrogen adsorbed on the surface of the copper, which is known as the Brunauer-Emmett-Teller (BET) method $^{8}$, using a NOVA2200e apparatus (Quantachrome Instruments, USA).

\section{RESULTS AND DISCUSSION}

3.1 Scanning Electron Microscopy (SEM)

Figure 1 shows an SEM image of the blended particles of copper and cellulose prepared by the high-speed ball milling for 3 hours. The sizes of these particles ranged from $4.7 \mu \mathrm{m}$ to $58.6 \mu \mathrm{m}$, which average out to $18.8(11.2) \mu \mathrm{m}$ calculated from randomly selected 100 particles; the size of a particle is the average of the longest and shortest length of the particle and the value in parenthesis represents the standard deviation.

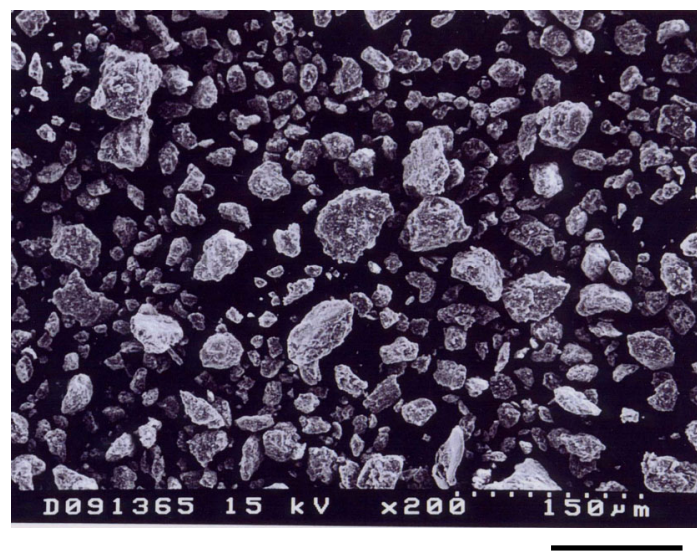

$100 \mu \mathrm{m}$

Fig.1 SEM image of cellulose/copper blend prepared by the high speed ball milling for 3 hour
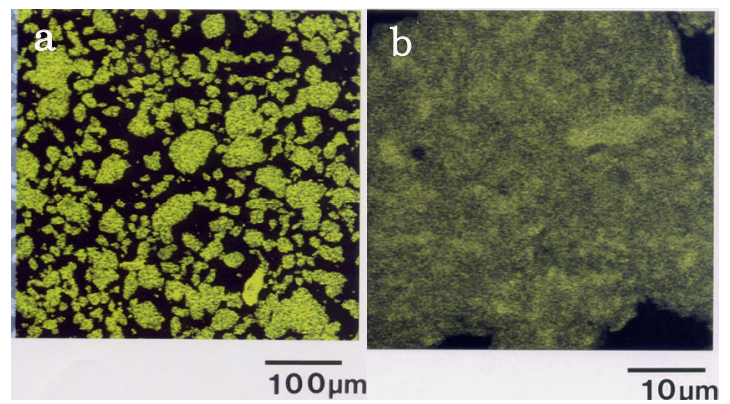

Fig.2 Distribution maps of cellulose / copper blend observed with energy dispersive X-ray micro analyzer.

Figure 2a shows the distribution map of copper for the same image shown in Figure 1. Yellow indicates domains where copper exists. The yellow regions had the same shapes as the particles shown in Figure 1, meaning that 
copper fragments were distributed homogeneously in the particles. A magnified view of the copper distribution shown in Figure $2 \mathrm{~b}$ also indicates the homogeneous existence of copper. However, the resolution of the apparatus used for this copper mapping was not so high (about $1 \mu \mathrm{m}$ ) as to examine the blended state of copper and cellulose.

\subsection{Scanning Probe Microscopy (SPM)}

The copper/cellulose blend materials were imaged by SPM using the surface potential microscopic mode. This mode can distinguish between copper and cellulose because the electrostatic potentials of the two materials are very different. In the SPM image (Figure 3), the black and white parts indicate copper and cellulose, respectively. The copper fragments were dispersed in the cellulose matrix in the sub-micron and nanometer scale. During the high-speed milling, the impact of heavy ceramic balls possibly caused amalgamations of copper and cellulose followed by breakdown of the fused pieces. It seemed reasonable to assume that the countless repeats of amalgamations and breakdowns mixed the copper/cellulose blend materials together on a sub-micron scale. The highest surface energy of cellulose $(45 \mathrm{mN} / \mathrm{m})^{9}$ among polymers probably provides strong contact between cellulose and copper. If it were not for the strong interaction between cellulose and copper, the two materials would be divided along the interface and would not be blended in this small scale. This is the main reason to use cellulose as the first polymer material for this study. When the copper alone was treated by the high speed ball milling for 3 hours, the size of the copper particles did not reduce, but became much larger, up to $0.2-0.5 \mathrm{~mm}$, with a scale-like shape. This is probably due to the ductility of copper, which makes the copper powders stick together to form larger granules rather than being crushed to small pieces.

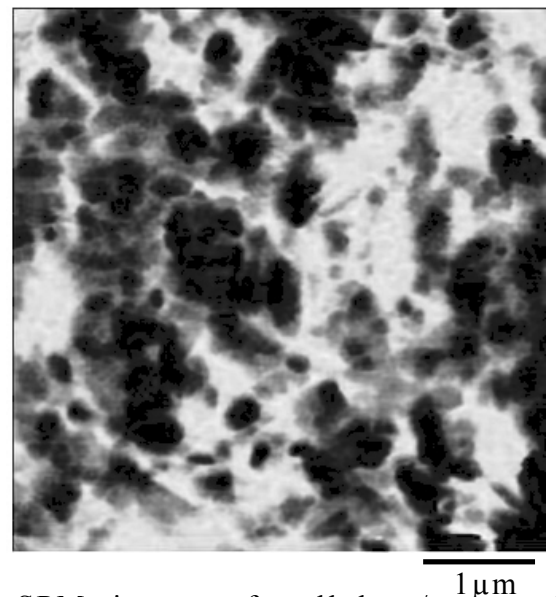

Fig. 3 SPM image of cellulose/copper blend using surface potential microscopic mode.

3.2 Structural change during high-speed ball milling
Figure 4 shows changes in the $X$-ray diffraction patterns of cellulose during high-speed ball milling. Clear diffraction peaks from the (1-10), (110) and (200) crystal planes of the cellulose I crystal from the lower diffraction angle were observed for the original cellulose (Figure 4(a)). The diffraction peaks abruptly decreased after a 10 minute treatment (Figure 4(b)) leading to one broad peak after 2-3 hours treatment (Figure 4(d,e). In spite of the drastic change in crystallinity, decreases in molecular weight $\left(\mathrm{M}_{\mathrm{W}}\right)$ were not very high, i.e., 36,700 for original cellulose, 26,100 for 2 hour ball milling and 26,200 for 3 hour ball milling. On the other hand, the X-ray diffraction pattern of copper remained unchanged even for the 3 hour treatment. The diffraction peak at $2 \theta=$ $28.45^{\circ}$ is from $\mathrm{SiO}_{2}$ used as an internal reference.

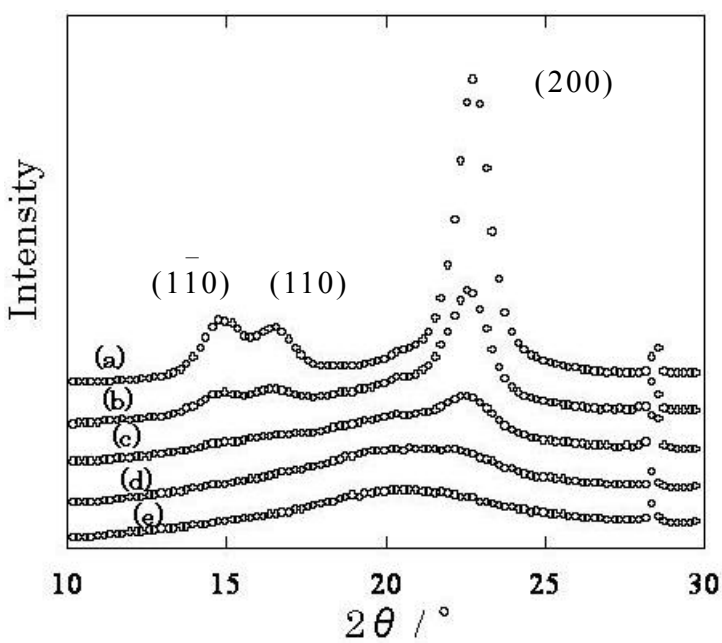

Fig.4 Changes in X-ray diffraction patterns of cellulose during high speed ball milling: (a), original cellulose; (b), ball milled for $10 \mathrm{~min}$; (c), $30 \mathrm{~min}$; (d), 2 hr.; (e), $3 \mathrm{hr}$.

\subsection{Porous Copper}

When cellulose in the blend materials was removed by firing or enzyme decomposition, porous copper could be obtained. Figure 5 shows an SEM image of porous copper prepared by firing at $600^{\circ} \mathrm{C}$ for 3 hours. The starting blend material subjected to firing was the same one shown in Figures $1-3$. The surface area of the fired particles was $9.60 \mathrm{~m}^{2} / \mathrm{g}$, which was much higher than that of the starting copper powder $\left(0.3 \mathrm{~m}^{2} / \mathrm{g}\right)$ prepared by an atomizing method. Every copper fragment seemed to be connected by sintering. It is not surprising that sintering took place at a far lower temperature $\left(600^{\circ} \mathrm{C}\right)$ than the melting point of copper $\left(1085^{\circ} \mathrm{C}\right)$, because the melting temperature decreases with decreasing size; for instance, the melting point of bulk gold $\left(1064^{\circ} \mathrm{C}\right)$ decreases to $330^{\circ} \mathrm{C}$ for gold particles with a diameter of 2 $\mathrm{nm}^{10}$. This suggests that the copper fragments in the blend materials include very small ones. 


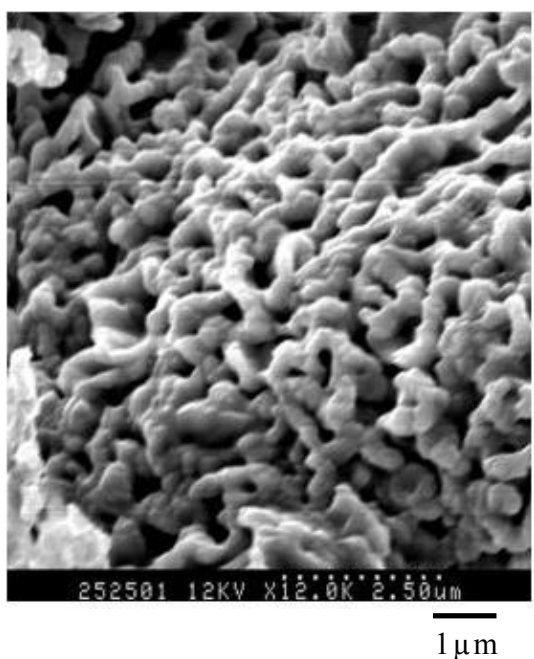

Fig.5 The SEM image of porous copper prepared by firing at $600^{\circ} \mathrm{C}$ for 3 hour in order to remove cellulose from the blend.

Porous copper can also be prepared by another method. Figure 6 shows the porous copper obtained from the same blend particles as the ones mentioned above using enzymatic decomposition of cellulose. The surface area of these particles was $18.32 \mathrm{~m}^{2} / \mathrm{g}$, which was higher than that of the fired particles (9.60 $\mathrm{m}^{2} / \mathrm{g}$ ), probably because no sintering occurred during the enzyme treatment. The shape and the size of the copper fragments were almost the same as those shown in Figure 3, indicating that Figure 6 shows the skeleton of the copper buried in the cellulose matrix.

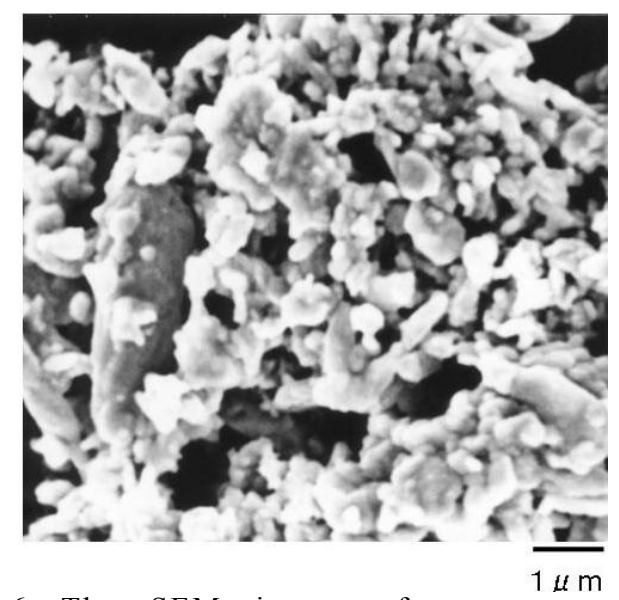

Fig.6 The SEM image of porous copper prepared by enzymatic decomposition of cellulose.

A closer look at Figure 6 shows some junction points present among the copper fragments. In addition to this, the blending mechanism, amalgamations and breakdowns, support the possibility that the copper fragments were not isolated from each other but rather were connected to each other at the junction points. Therefore, such a porous structure must prevent cohesion of the metal fragments to retain its effective surface area. Various nano-metals have a problem that needs to be solved, that is, cohesion of the particles decreases the apparent surface area resulting in a decrease in the efficiency for their fine use. Therefore, if the porous metals prepared by this method are applied as catalysts or electrodes, a high efficiency can be expected and anticipated.

\section{REFERENCES}

1. T. Endo, R. Kitagawa, F. Zhang, T. Hirotsu and J. Hosokawa, Chem. Lett., 1155(1999).

2. H. Kitagawa, M. Iseya, N. Kubo, T.Endo, K.Moriya and K.Okita, Woody Composition for Molding and Its Manufacturing Method, publication number (2002-225011), Japan, date of publication of application, 14/08/2002.

3. Y. Yamaguchi, T. Noguchi and K. Goto, Sen-i Gakkaishi (Journal of the Society of Fiber Science and Technology, Japan), 49, 124(1993). 4. Y.Nakamichi, Preparation of Polymer/Metal Cluster Composite; patent number (3062748), Japan, issue date 10/03/1999.

5. J.Hasegawa, M.Ito, K.Niihara, T.Nakayama, Method for Manufacturing Composite Material, publication number (2006-089523), Japan, date of publication of application, 06/04/2006.

6. R. Saito and K. Ishizu, Polymer, 33, 1099(1992).

7. M. Harada, K. Asakura, Y. Ueki and N. Toshima, J. Phys. Chem., 98, 2653(1994). 8. S. Brunaur, P.H. Emmett and E. Teller, $J$. Amer. Chem. Soc., 60, 309(1938).

9. "Polymer Handbook - Fourth Edition -", Ed. by J. Brandrup, E.H. Immergut, and E.A. Grulke, Wiley Interscience, (1999) p.VI 533.

10. P. Buffat and J. Borel, Physical Review A, 13, 2287-2298(1976).

(Received December 12, 2008;Accepted September 16, 2009) 